\title{
An Adaptive Timeslot Allocation Scheme for Wireless Body Area Networks
}

\author{
Bindu Bala \\ Department of Computer \\ Science and Engineering, \\ M.M.University,Mullana, \\ Ambala, Haryana
}

\author{
Monica Pandey \\ Department of Computer \\ Science and Engineering, \\ M.M.University,Mullana, \\ Ambala, Haryana
}

\author{
Devendra Prasad, PhD \\ Department of information \\ technology, \\ M.M.University, Mullana, \\ Ambala, Haryana, \\ India.
}

\begin{abstract}
With the rapid growth in specialized biomedical sensors, wireless communication and low power integrated circuits; the wireless sensor network has achieved a new milestone. IEEE 802.15.4 standard uses carrier sense multiple access with collision avoidance (CSMA-CA) medium access mechanism and supports star as well as peer-to-peer topologies. By using superframe structure, time slots can be allocated by the Coordinator to devices with time critical data. In IEEE 802.15.4 due to the low SNR (signal to noise ratio) carrier sensing is not reliable in UWB PHY, the "hidden Terminal problem" arises due to the use of CSMA-based random access control, and due to the presence of only one common contention based access period, the CAP part deprives the priority of life critical medical applications.
\end{abstract}

To overcome these limitations of IEEE 802.15.4, an adaptive timeslot allocation scheme based on precedence is proposed. In proposed scheme, Data traffic is divided into 2 priority classes and CAP is categorizes into 2 access phases. Collision probability and final throughput are influenced by the performance of CAP. CAP is divided into 2 sub-phases: phase- 1 , and phase-2. $\mathrm{C}_{1}$ represents first class priority, and $\mathrm{C}_{2}$ represents the second class priority. Second class priority includes both continuous and non continuous data.

\section{Keywords}

Superframe, backoff, overhearing.

\section{INTRODUCTION}

With the rapid growth in specialized biomedical sensors, wireless communication and low power integrated circuits; the wireless sensor network has achieved a new milestone. Wireless body area network (WBAN) is simply an application of wireless sensor network (WSN) [1] [2]. It is similar to WSN, the features that differentiate between the two are, number of nodes, working area, power consumption by nodes, and protocols used for efficient communication. Wireless body area network is a network formed by the wireless sensors either on or inside patients' body. Sensors implanted on or inside the body are also named as nodes [3]. These sensors sense, store and send the information to the doctor using internet service. It is an interdisciplinary area which could allow continuous and in expensive health monitoring with real time updates of medical records through the internet. It is a wireless wearable system used for the active recognition and vital sign monitoring. The objective of this application is to remotely capture vital signs by simultaneously monitoring human physical activities. This correlation of vital signs with physical activity will have a tremendous impact on developing inexpensive solutions for remote health monitoring and diagnosis in the developing world. Also health monitoring for health risk analysis in long term can also be enabled by this correlation of physical activity with vital signs. The main aim of this application is to make BAN non invasive and non intrusive wireless health monitoring network [3]. WBAN is capable of recognizing human activities by using the information collected by all the nodes implanted on the body.

According to a survey conducted by an organization, by 2030 there will be 71 million older persons. At present the number is half. WBAN is a boon for aged patients. It facilitate the monitoring of aged patients lying either in old age homes or alone at their homes requiring continuous monitoring of body parameters while they carry out their daily routines. Continuous monitoring is necessary whenever data collected by the nodes shows abnormal changes, it is necessary to take quick desired actions, so that the precious lives can be saved. WBAN has wide range of applications [4].IEEE 802.15.4 MAC protocol can work, either in beacon enabled mode or in non-beacon enabled mode. To achieve reliable MAC control, beacon enabled mode is used. A superframe contains an active and inactive portion.

The active period is mandatory and the inactive period is optional. The inactive part is used for low rate and low-power considerations. The active part is further divided into three parts: a beacon, contention access period (CAP), and contention free period (CFP). Several limitations of this protocol are revealed, when used in WBAN scenario. First one is, due to the low SNR (signal to noise ratio) carrier sensing is not reliable in UWB PHY. The "hidden Terminal problem" arises in IEEE 802.15.4 due to the use of CSMAbased random access control. Second limitation is the presence of only one common contention based access period, the CAP part, which deprives the priority of life critical medical applications.

Some schemes are proposed that uses parameter-based priority control. Regardless of this, soft control cannot guarantee the priority of the medical applications because some self centered nodes can always use short contention window and back-off delay without being noticed by the master node. Third limitation is that this protocol does not consider the energy efficiency of the master node. The rest of the paper is divided into the following sections. II section describes the literature work related to the paper. Proposed scheme and related algorithms has been described in the next section. Network model is described in section IV. Section V evaluates the results. Finally the conclusion has been drawn in VI section.

\section{RELATED WORK}

Authors in [5] proposed a new protocol to satisfy both medical and CE applications. As in WBAN both the applications have different requirements and hence it is a new challenge for the WBAN. Addressing this problem, a priorityguaranteed MAC protocol is proposed in this paper. In this protocol, data channels are separated from control channels to 
support collision free high data rate communication for CE applications. Priority-specific control channels are adopted to provide priority guarantee to life-critical medical applications. Traffic-specific data channels are deployed to improve resource efficiency and latency performance.

Moreover, in order to further minimize energy consumption and access latency, an asynchronous wakeup trigger mode is proposed as an enhancement to the priority traffic. The customized priority-guaranteed MAC satisfies the service requirements of WBAN by making tradeoff among the performances of different applications. Generally devices in WBAN are deployed in less dense manner unlike WSN, but exceptions may exist in which devices may be deployed densely. Compared with WSN, however, WBAN operates in a Centralized manner, and thus, contention complexity on contention-based phase of WBAN may be larger than WSN.

Algorithm proposed by the authors in [6] proposes a novel channel access algorithm for contention based MAC protocol in WBANs. The proposed algorithm uses four ideas: categorizing packet into 4-levels, defining a delay threshold for each level of packet, dividing CAP into sub-phases by threshold, and calculating offsets to decide the length of divided sub-phase.

By classifying categories of packets into 4-level and dividing CAP into sub-phases, proposed algorithm can disperse contention complexity and reduce power consumption The proposed algorithm classifies the contention-based access phase into 4-levels by the priority of packet that is defined in WBAN draft document, and divides the contention-based access phase into sub-period by calculated offset. Extensive simulation results show that the proposed algorithm achieves low complexity such as reduced collisions, low power consumption, and high channel utilization compared with the IEEE 802.15.6 baseline MAC protocol [7].

The authors in [8] introduce priority based Binary Exponential Backoff algorithm (PB-BEB). The IEEE 802.15.4 [9] [10] standard utilizes the CSMA-CA mechanism to control nodes' access to the shared wireless communication medium. CSMA-CA implements the Binary Exponential Backoff (BEB) algorithm by which a node refrains from sending any packet before the expiry of its backoff period. After that, the node is required to sense the medium for two successive time slots to assert that the medium is clear from any ongoing transmissions (this is referred to as Clear Channel Assessment (CCA)).

Upon finding the medium busy, the node doubles its backoff period and repeats that process. While effective in reducing the likelihood of collisions, this approach takes no measures to preserve the priorities among the nodes contending to access the medium. Author proposes the Priority-Based BEB (PB-BEB) algorithm in which we enhance BEB such that nodes' priority is preserved. This algorithm is used to manage the contention among the nodes attempting to access the wireless medium. The problem with BEB is that it treats all the nodes equally, without giving any consideration, to the repeated channel access failures or transmission failures experienced by some nodes.

The Priority-Based $\mathrm{BEB}$ is designed to fill that hole in the design of BEB. In PB-BEB, the number of CCA is controlled probabilistically according to the collision level over the communication medium. As the node finds the medium busy at a certain $\mathrm{CCA}$, beyond the mandatory $C C A \mathrm{~s}$ of $\mathrm{BEB}$, the node will backoff. At the end of the last backoff, the node will start its clear channel assessment at exactly the last CCA where it stopped. This means that the node that has been experiencing multiple back-offs while trying to send a packet is given a higher priority to access the medium than those nodes that started their contention at a later time. PB-BEB shows superior performance, over BEB, in terms channel utilization, reliability, power wasted in collisions, and channel collision time.

\section{PROPOSED SCHEME}

\subsection{Data Classification Based on Priority}

Medical and CE (consumer electronics) signals are the main traffic in the network. Some irregular emergency signal is also part of traffic in the network. Emergency traffic is directly related to the life of users (patients), hence the emergency signal is most important among all the signals in the traffic. Whenever emergency signals occur within the traffic, these signals should be transmitted immediately to the desired location.

Table: 1 Classification of Data

\begin{tabular}{|c|c|c|c|}
\hline Priority & $\begin{array}{l}\text { Representative } \\
\text { Symbols }\end{array}$ & Data Type & Examples \\
\hline 1 & $\mathrm{C} 1$ & Emergency Alam & Emergency Vital Signals \\
\hline 2 & $\mathrm{C} 2$ & $\begin{array}{c}\text { Normal data (continuous } \\
\text { \& non continuous) }\end{array}$ & $\begin{array}{c}\text { Temperature/Blood Pressure } \\
\text { EEG/EMG }\end{array}$ \\
\hline
\end{tabular}

Immediate transmission of these signals may save the life of the patient. The remaining signals (normal data) are considered to be less important and hence transmitted using the normal transmission procedure.

The proposed scheme classifies the data into two priorities as shown in Table 1.

\subsection{Prioritize Timeslot Allocation Scheme}

In this scheme, Data traffic is divided into 2 priority classes and CAP is categorizes into 2 access phases. Collision probability and final throughput are influenced by the performance of CAP. CAP is divided into 2 sub-phases: phase-1, and phase-2. $\mathrm{C}_{1}$ represents first class priority, and $\mathrm{C}_{2}$ represents the second class priority. Second class priority includes both continuous and non continuous data.

Nodes that transmit $\mathrm{C}_{1}$ data can access the channel through phase-1, and phase-2. Nodes that transmit $\mathrm{C}_{2}$ data can access the channel through phase- 2 only. Nodes having lowest priority, i.e. nodes transmitting $\mathrm{C}_{2}$ data can only use phase- 2 to access the medium. It means that phase- 1 can be used only for transmitting emergency data; phase- 2 can be used to transmit emergency as well as all types of medical data.

Even if $\mathrm{C}_{1}$ exists or not, phase- 1 always exists and given first timeslot of CAP by the coordinator. Length of phase- 1 is by default one timeslot. Figure 2 shows the superframe structure of the proposed scheme. The number of nodes that transmit $\mathrm{C}_{2}$ will change with the addition and removal of nodes from the network. When a new node wants to join in the network, the coordinator handles the newcomer nodes.

\section{Notations used in the proposed scheme:}

$\mathbf{N}$ : Total number of active nodes in the network

$\mathbf{C}_{1}$ : First priority class data (emergency data)

$\mathbf{C}_{2}$ : Second priority class data (normal data) 
$\mathbf{n C}_{\mathbf{1}}$ : Number of nodes that transmit $\mathrm{C}_{1}$

$\mathbf{n C}_{2}$ : Number of nodes that transmit $\mathbf{C}_{2}$.

$l$ : Length of CAP (unit is timeslot)

$\boldsymbol{l}_{\boldsymbol{l}}$ : Length of Phase-2 (unit is timeslot)

If $l_{1}$ 's value is fixed, the timeslot in phase- 2 will not be utilized properly (underutilized) when the nodes in network that transmit $\mathrm{C}_{2}$ are less.

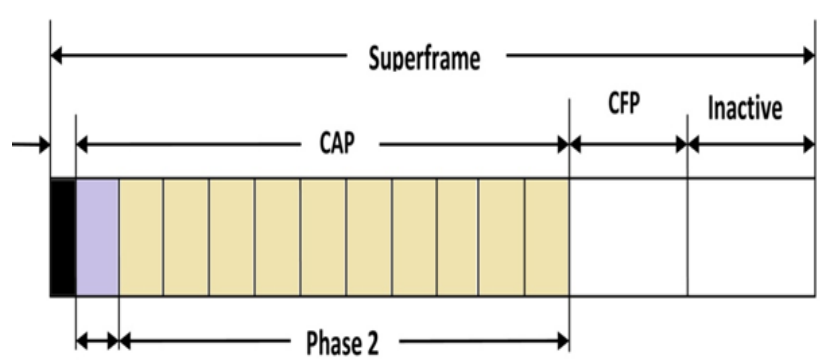

Phase 1

Figure: 1 Superframe Structure of Proposed Scheme

Hence, in order to avoid the wastage of slots, $l_{1}$ is calculated by the coordinator and changes dynamically with equation 1

$$
l_{1}=l-1
$$

In IEEE 802.15.4 standard supports only one type of data.

The Coordinator learns the information of each node's priority to update the current number of $\mathrm{nC}_{2}$ in the network.

To join the network node sends an Association Request Command in CAP to the Coordinator and inform about it own priority. The Coordinator computes the number of nodes that transmits $\mathrm{C}_{2}$ type of data and informs all nodes about the modification of $l_{1}$ by sending beacon at the beginning of current super-frame.

In this way the proposed scheme achieves scalability to cope with the dynamic network.

\subsection{Data Transfer Procedure}

The superframe structure contains CAP and CFP. In IEEE802.15.4, CAP is suitable for the transfer of command message and short message, CFP is used for the transfer of continuous data. Keeping this concept in mind, two types of data (emergency and normal data), are successfully transmitted over the communication channel without collision.

Regulation procedure of the transmission is as follows:

- $\mathrm{C}_{1}$ : After gaining access to the communication channel in CAP, $\mathrm{C}_{1}$ data transmitted immediately.

- $\quad \mathrm{C}_{2}$ : When nodes have normal data to transmit, sends GTS request command in CAP for GTS allocation, transmit $\mathrm{C}_{2}$ in allocated GTS in next superframe.

Figure 2 shows the data transfer procedure in IEEE 802.15.4 and the proposed scheme. Under proposed scheme $\mathrm{C}_{2}$ has lower priority than $\mathrm{C}_{1}$, thus whenever $\mathrm{C}_{1}$ is present, priority is given to it. $\mathrm{C}_{1}$ can be directly sent in CAP unlike $\mathrm{C}_{2}$. Compared to IEEE802.15.4, we can see that in proposed scheme most short messages and command messages delivered in CAP. The normal traffic transmitted in CFP of the next super-frame.

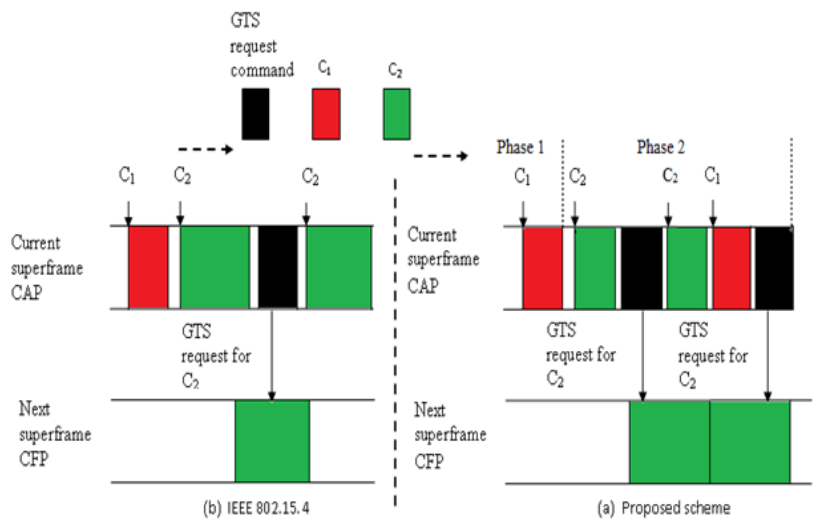

Figure 2: Comparison between IEEE 802.15.4 and the Proposed Scheme.

\subsection{Algorithm Realization}

Algorithm realization is divided into two parts: algorithm for a coordinator and another is algorithm for the sensor/nodes.

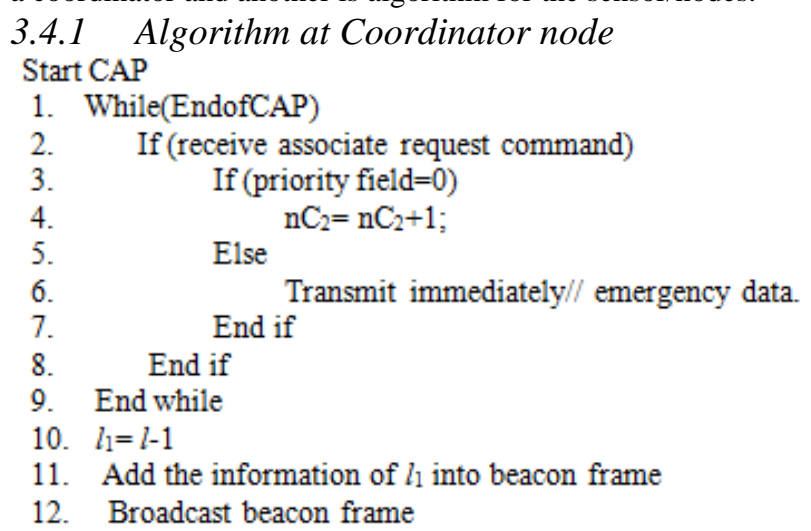

\subsubsection{Algorithm for sensor Start CAP}

1. Receive beacon from the coordinator

2. If (node is newcomer) 3. Send (associate request command frame)

4. End if

If (node is already in the network)

6. If (data packet arrives from upper layer)

7. If $\left(\mathrm{C}_{1}\right)$

8. Send $\left(\mathrm{C}_{1}\right)$

9. End if

10. If $\left(\mathrm{C}_{2}\right)$

11. Send (GTS request command)

12. End if

13. End if

14. End if

15. End of CAP

\section{NETWORK MODEL}

The Figure 3 shows the network model of the proposed scheme. The Star topology is selected for the following reason 


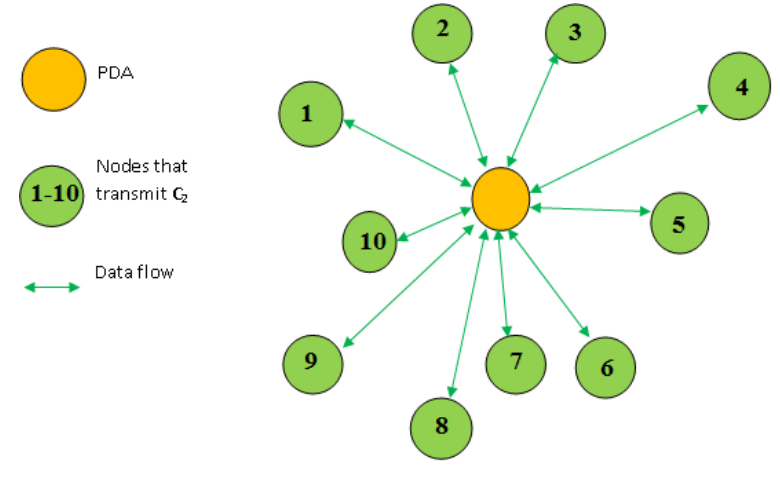

Figure 3: Star topology

- Simplicity in functioning \& easy to manage.

- Best suitable for small networks.

This topology reduces the chances of network failure by connecting all the nodes to the central device. Each node is connected with the central node (PDA/coordinator). The Coordinator maintains the record of each node and calculates the timeslot for each node by specified algorithm. All traffics in the network passes through the coordinator as all nodes report to the Coordinator. To join the network nodes sends association request command to the Coordinator, along with its "priority" level. The Coordinator analyses this field and on the basis of type of priority, it allocates the time slots to the requesting nodes.

In the network, only uplink data flow is considered. Since the proposed scheme focuses on the service differentiation within CAP in beacon enabled superframe mode, the active portion contains only CAP part, CFP is not included in active portion. Each node in the network uses slotted CSMA/CA to access the communication channel within CAP.

\subsection{Access Probability}

Access probability that a sensor node successfully access the channel with maximum back-off number 5 is given by equation (2).

$$
\mathrm{S}=\sum_{\mathrm{k}=1}^{5} p(1-p)^{\mathrm{k}-1}
$$

Where $p$ is the probability that at the end of backoff interval, the channel is clear: With $\mathrm{n}$ sensors in a network, the probability of clear channel in a CCA period is given by equation (3).

$$
p=(1-q)^{\mathrm{n}-1}
$$

Where, $q$ is the probability that a sensor node transmits in a CCA period and calculated using equation (4). The value of the fixed time period for each node is related to the length of the access phase in CAP.

$q$ $=\frac{\text { average channel occupation time of a node in a fixed time period }}{\text { fixed time period }}$
The value of $q$ can be calculated if the average channel occupation time of a node in a fixed time period is known.

\subsection{Transmission Time and Energy Efficiency}

Transmission time is the amount of time taken by the message to reach its destination from its source. In a digital message, it is the time between the first bit, leaving the source and the last bit arriving at the destination. The time required by a message depends on the size of the message and the bandwidth of the channel. In MAC protocol design energy efficiency is another key measurement. Energy efficiency depends on the duration of sensor's transceiver state. The four states of sensor node are as:

- Sleep

- Idle

- $\quad$ Receive $\left(\mathrm{R}_{\mathrm{x}}\right)$

- Transmit $\left(\mathrm{T}_{\mathrm{x}}\right)$

\section{SIMULATION AND RESULT}

\section{ANALYSIS}

The proposed scheme is simulated in MATLAB and the result is analyzed and compared with IEEE 802.15.4 standard. Simulation parameters are given in table 2.

Table 2: Simulation parameters

\begin{tabular}{|l|l|}
\hline Parameter & Value \\
\hline$P_{T x}$ & $36.5 \mathrm{mw}$ \\
\hline$P_{R x}$ & $41.4 \mathrm{mw}$ \\
\hline$P_{\text {Idle }}$ & $712 \mu \mathrm{w}$ \\
\hline Channel Rate & $250 \mathrm{kbps}$ \\
\hline Symbol times & $16 \mu \mathrm{s}$ \\
\hline Superframe duration & $122.88 \mathrm{~ms}$ \\
\hline aUnitBackoffPeriod & 20 symbols \\
\hline macBeaconOrder $(\mathrm{BO})$ & 3 \\
\hline macSuperframeOrder $(\mathrm{SO})$ & 0 \\
\hline macMaxCSMABackoffs & 5 \\
\hline macMinBE & 3 \\
\hline macMaxBE & 5 \\
\hline$T_{\text {beacon }}$ & $0.36 \mathrm{~ms}$ \\
\hline
\end{tabular}

\subsection{Access probability}

Access probability of the proposed scheme is compared with IEEE 802.15.4 in Figure 4. We can observe that as the number of nodes increases the access probability decreases and in all case the (i.e. any type of data) channel access probability of the proposed scheme is higher than the IEEE 802.15.4 standard. 


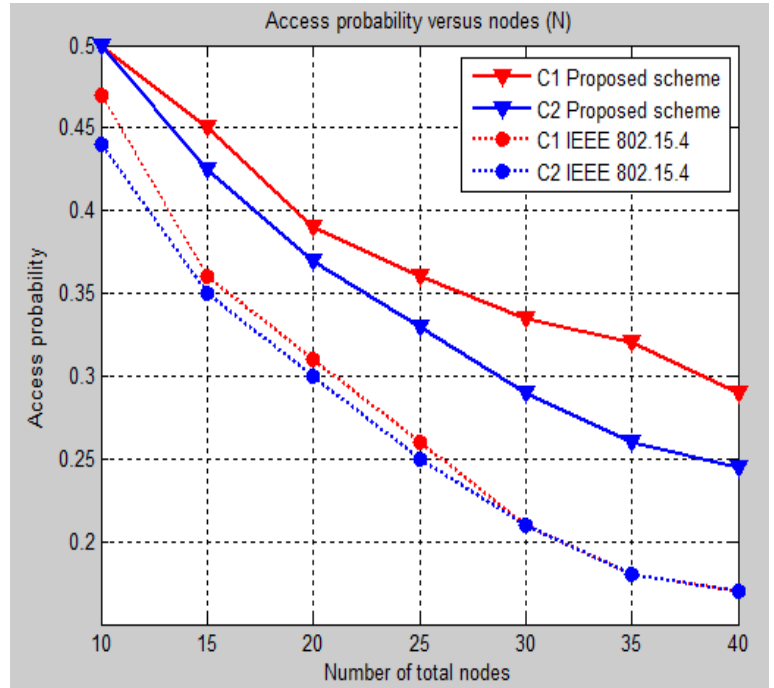

Figure 4: Comparison of IEEE 802.15.4 and the proposed scheme, based on access probability.

\subsection{Average energy consumption}

Figure 5 represent the average energy consumption in IEEE 802.15.4 and the proposed scheme. From Figure 5 it is clear that the as the number of nodes increases, average energy consumption also increases but the average energy consumption in the proposed scheme is always lower than IEEE 802.15.4 standard.

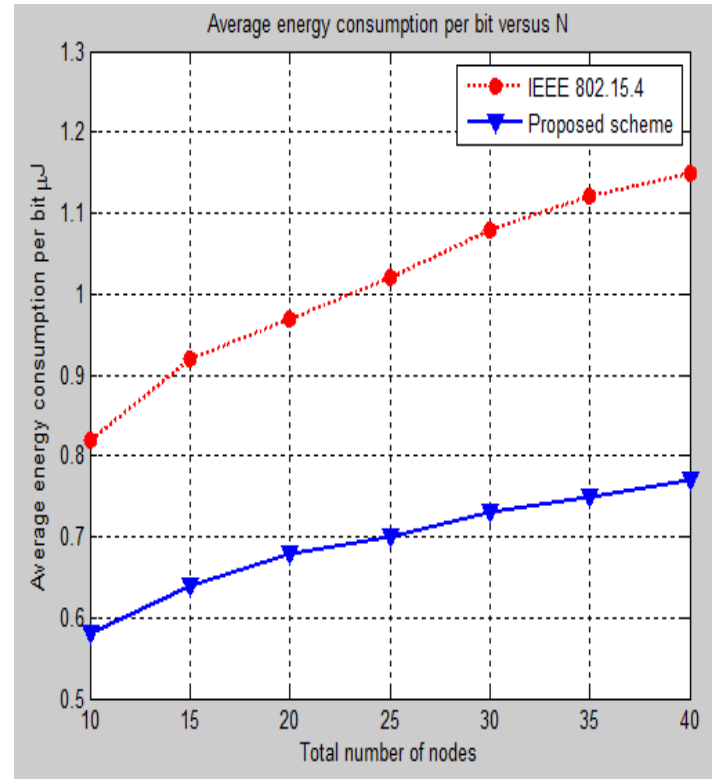

Figure 5: Total Nodes (N) Vs Average Energy Consumption per Bit

\subsection{Transmission Time}

The proposed scheme is compared with IEEE 802.15.4 standard on transmission time. The transmission time obtained for $\mathrm{C}_{1}$ and $\mathrm{C}_{2}$ is represented in the Figure 6. It is clear from Figure 6 that data transmitted using proposed scheme, takes less time as compared to IEEE 802.15.4.

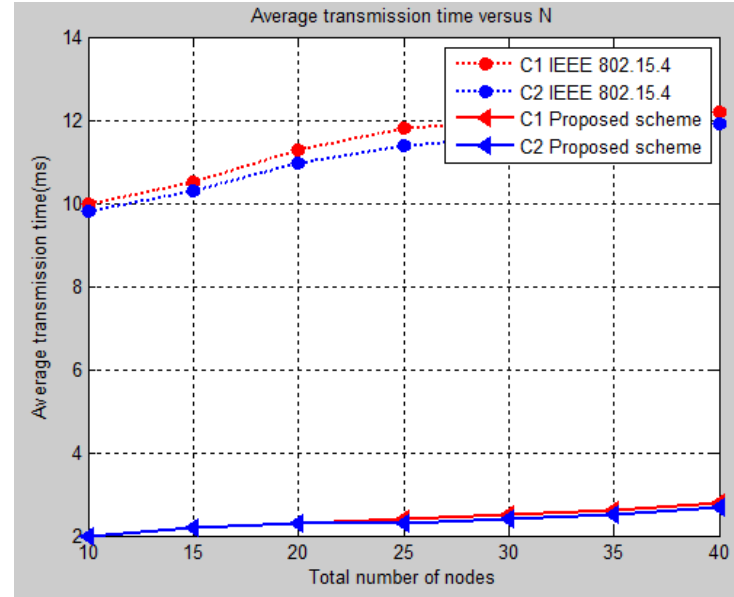

Figure 6: Comparison of IEEE 802.15.4 and the proposed scheme, based on transmission time (ms)

\section{CONCLUSION}

In existing protocol (IEEE 802.15.4) all nodes treated in same way, even if some nodes have emergency data to transmit. In this article a Time Slot Allocation scheme is developed in which special attention is given to the emergency data and transmitted immediately whereas normal data can be delayed. If more nodes are added to the existing network, the energy consumption in IEEE 802.15.4 rise hence network life decreases which is not a favorable condition. The proposed scheme consumes less energy as compared to IEEE 802.15.4 standard to transfer the data. By avoiding collision, reducing idle listening and overhearing the proposed scheme decreases energy consumption to some extent.

\section{REFERENCES}

[1] Beom Seok Kim and Jinsung Cho,"A Novel PriorityBased Channel Access Algorithm for Contention-based MAC Protocol in WBANs". Proceedings of the 6th International Conference on Ubiquitous Information Management and Communication (ICUIMC), New York USA, 2012-02-20, pp.1-5.

[2] Sai Anand Gopalan, Dong-Hyun Kim, Jae-Wook Nah, and Jong-Tae Park, "A Survey on Power-Efficient Mac Protocols For Wireless Body Area Network", Proceedings of IC-BNMT201 0, pp. 1230-1234.

[3] Mounib Khanafer, Mouhcine Guennoun, Hussein T. Mouftah "Priority-Based CCA Periods for Efficient and Reliable Communications in Wireless Sensor Networks “, scientific research, 2012, pp.45-51.

[4] S. A. Desai, P. H. Zope, S. R. Suralkar," A Review on IEEE 802.15.4- Standard for Wireless Body Network", International Journal of Innovative Research in Science, Engineering and Technology ,May 2013, pp. 1477-1486.

[5] Nicholas F. Timmons, William G. Scanlon. Analysis of the Performance of IEEE 802.15.4 for Medical Sensor Body Area Networking", Sensor and Ad Hoc Communications and Networks, IEEE SECON, First Annual IEEE Communications Society Conference, 4-7 Oct. 2004, pp no. 16- 24. 